Article

\title{
Toward Super-Resolution Imaging at Green Wavelengths Employing Stratified Metal-Insulator Metamaterials
}

\author{
Masanobu Iwanaga * \\ National Institute for Materials Science (NIMS), 1-1 Namiki, Tsukuba 305-0044, Japan; \\ E-Mail: iwanaga.masanobu@nims.go.jp
}

Received: 30 March 2015 / Accepted: 2 May 2015 / Published: 7 May 2015

\begin{abstract}
Metamaterials (MMs) are subwavelength-structured materials that have been rapidly developed in this century and have various potentials to realize novel phenomena, such as negative refraction, cloaking and super-resolution. Theoretical proposals for super-resolution image transfer using metallic thin films were experimentally demonstrated at ultraviolet and violet wavelengths from 365 to $405 \mathrm{~nm}$. However, the most preferred wavelengths of optical imaging are green wavelengths around $500 \mathrm{~nm}$, because optical microscopy is most extensively exploited in the area of biotechnology. In order to make the super-resolution techniques using MMs more practical, we propose the design of a stratified metal-insulator MM that has super-resolution image transfer modes at green wavelengths, which we here call hyper modes. The design assumed only $\mathrm{Ag}$ and $\mathrm{SiO}_{2}$ as constituent materials and was found employing Bloch-state analysis, which is based on a rigorous transfer-matrix method for the metal-insulator MMs. It is numerically substantiated that the designed stratified metal-insulator metamaterial (SMIM) is capable of forming super-resolution images at the green wavelengths, and optical loss reduction is also studied. We discuss the results derived by the Bloch-state analysis and by effective medium models usually used for the metal-insulator MMs and show that the Bloch-state analysis is more suitable to reproduce the experimental data.
\end{abstract}

Keywords: metamaterials; super-resolution imaging; metal-insulator multilayers; visible imaging; dispersion analysis; Bloch states 


\section{Introduction}

Super-resolution (SR) image transfer in metamaterials (MMs) was stimulated by a paper on SR imaging by a silver slab [1] and has been developed theoretically [2-4] and experimentally [5-9]. The MMs for SR imaging are stratified metal-insulator metamaterials (SMIMs) and one of the simplest MMs in structure, as illustrated in Figure 1. In addition to the SR imaging, it was found that the SMIMs enable intriguing phenomena, such as isotropic negative refraction [10,11]. The experimental demonstrations on the SR imaging and the negative refraction were mostly conducted at the ultraviolet (UV) to violet wavelength range (365 to $405 \mathrm{~nm}$ ), where SMIMs of finite thickness show high transmittance (T) up to tens of a percent.

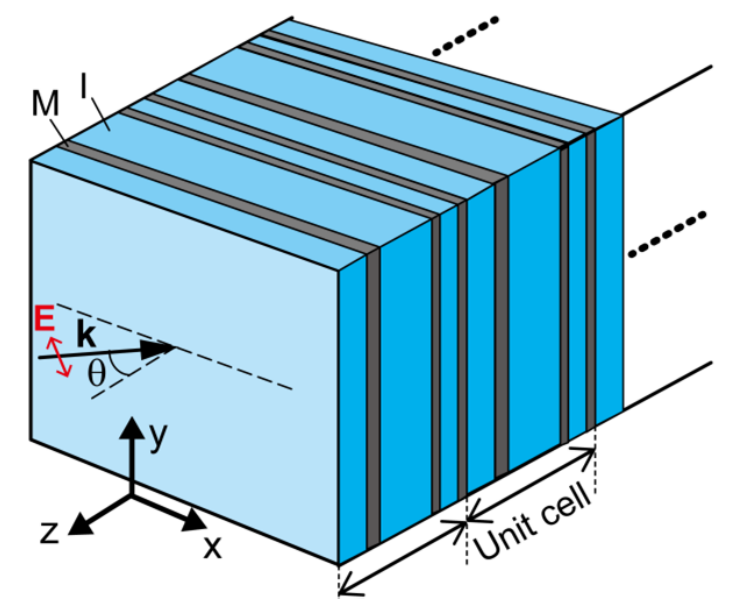

Figure 1. Schematic of stratified metal-insulator metamaterials (SMIMs) of a complex unit cell. As an example, a SMIM of a six-layer unit cell is illustrated. Gray denotes metal (M) and pale blue insulator (I). The optical configuration of incidence is also shown with the wavevector $\mathbf{k}$, the E-field vector $\mathbf{E}$ and the angle $\theta$.

One of the issues in SMIMs is to move the transmission band associated with the SR imaging to desired wavelength ranges. To date, it was reported that SMIMs, including insulators of a high refractive index of about 2.4 [8,10,11], have a transmission band at a longer wavelength range than SMIMs of a low refractive index of about 1.5. However, it has not been reported that SMIMs works at about 500 $\mathrm{nm}$ or green wavelengths, even if the high-index insulators are incorporated. The best wavelengths for imaging of biological objects are the green wavelengths. Therefore, to make the SR optical imaging more practical, it is highly desirable to move the working wavelengths to the green wavelengths, In addition, well-known low-index insulators, such as $\mathrm{SiO}_{2}$, have an advantage in making the fabrication procedure more feasible and low cost, because they are widely used and common materials. Designs to meet the requirements above have not been presented to date.

When considering further advances in SMIMs application, there is a fundamental issue. SMIMs have been conventionally explained, assuming an effective medium model; that is, SMIMs are assumed to be uniaxial media and to be described using the effective permittivity of $\varepsilon_{\|}$and $\varepsilon_{\perp}$, where the symbols $\|$ and $\perp$ denote parallel and perpendicular to the principal axis in the uniaxial media, respectively. It has been furthermore assumed that the effective parameters $\varepsilon_{\|}$and $\varepsilon_{\perp}$ are local and independent of wavevector $\mathbf{k}$ of electromagnetic (EM) waves in SMIMs. These assumptions lead to so-called hyperbolic 
dispersion $[3,4,12-16]$ in the $\left(k_{x}, k_{z}\right)$ plane in the optical configuration of Figure 1. However, the validity of the model has not been examined in most cases. Indeed, there is a limitation of the effective medium model for SMIMs concerning wavelength range [17]. Based on a rigorous transfer matrix [18,19], a more thorough theoretical analysis employing photonic Bloch states was explored for multilayered metal-insulator structures [20-25]. Among the reports on the Bloch-state analysis, a realistic procedure, which directly corresponds to the experiment, incorporating the actual permittivity of metal and the imaginary part of the Bloch eigenvalues, was limited to [21,22].

In this paper, we propose concrete designs of SMIMs with a diffraction-suppressing mode at blue and green wavelengths; we here call the modes hyper modes. The designs require Ag and only a low-index insulator, such as $\mathrm{SiO}_{2}$, which has refractive index of 1.46 in the visible spectrum. The hyper modes are explicitly derived employing the Bloch-state analysis [21,22]. The SMIMs with hyper modes were found by a structural search that was a combination of the random generation of unit cells in SMIMs and of refined optimization of a selected good unit cell. The properties of light propagation in SMIMs are quantitatively analyzed and are clarified without relying on the effective medium model that is often assumed for SMIMs. The deeply subwavelength image transfer by the SMIMs of a low-index insulator is directly examined, and the loss reduction in SMIMs with the gain insulator is also studied. From the viewpoint of the Bloch states, we also discuss other experimental SMIMs $[6,8,11]$ and reach a result that the hyperbolic dispersions assumed in the reports $[3,4,12-15]$ are limited to a wavenumber range around the origin of the dispersion diagram. The result is consistent with recent theoretical implications [24,25].

\section{Designs of Complex Unit Cells in SMIMs}

Figure 1 depicts a schematic of the SMIM of a complex unit cell; a SMIM of a six-layer unit cell is illustrated to show the incident configuration. The coordinate system is defined such that the $x y$ plane is parallel to the metal (M) and insulator (I) layers, which are shown with gray and pale blue, respectively, and the $z$ axis is parallel to the stacked direction. The $M$ and I layers are assumed to spread enough along the $x y$ plane. The plane of incidence is set to be parallel to the $x z$ plane; the $p$-polarized incident plane wave is shown, and then the electric-field (E-field) vector $\mathbf{E}$ is parallel to the $x z$ plane. When incident light is $s$ polarized, the E-field vector satisfies with $\mathbf{E}|| y$.

\subsection{Four-Layer Unit Cell for Blue Wavelengths}

Figure 2a shows a schematic illustration of a SMIM of a four-layer unit cell of $\left(\mathrm{SiO}_{2} 50 \mathrm{~nm} / \mathrm{Ag} 25\right.$ $\mathrm{nm} / \mathrm{SiO}_{2} 80 \mathrm{~nm} / \mathrm{Ag} 25 \mathrm{~nm}$ ). A SiO 2 layer of $50 \mathrm{~nm}$ in thickness is exposed to the incident layer of air. The SMIM comprises 16 layers in total and has a $720-\mathrm{nm}$ thickness. A feature of the SMIM is the transmission window at $410 \mathrm{~nm}$. 
Measured and calculated T spectra under $p$ polarization are shown in Figure $2 \mathrm{~b}$ and $\mathrm{c}$, respectively. The $\mathrm{T}$ spectra are shown with an offset for clarity, represented in the top-to-bottom manner in accordance with incident angles $0^{\circ}$ to $60^{\circ}$. The measured T spectra show a quantitatively good agreement with the calculated ones for the wide incident angles. The specimen was made by ion-beam sputtering onto a $\mathrm{SiO}_{2}$ substrate; the thin-film growth enabled highly precise control of the thickness of each layer. The numerical calculation was implemented by using the scattering-matrix (S-matrix) algorithm [26] and by taking the measured permittivity of $\mathrm{Ag}$ [27] and the typical permittivity of $\mathrm{SiO}_{2}, 2.1316$, and air, 1.00054 .

Figure 2d presents the transmission Bloch band of the SMIM of the four-layer unit cell at transverse magnetic (TM) polarization, at which we set the E-field vector to be in the $x z$ plane. We mention that the TM polarization corresponds to $p$ polarization in the illuminating configuration of Figure 1 . The Bloch band is well defined in perfectly periodic SMIMs, which was determined by solving the following eigenvalue equation:

$$
\mathcal{T}\left(\begin{array}{l}
F^{+}\left(z_{0}\right) \\
F^{-}\left(z_{0}\right)
\end{array}\right)=\exp (i K d)\left(\begin{array}{l}
F^{+}\left(z_{0}\right) \\
F^{-}\left(z_{0}\right)
\end{array}\right)
$$

where $\mathcal{T}$ denotes the $2 \times 2$ transfer matrix for one periodicity $d, F^{ \pm}$is the EM-field component propagating for $\pm z$ directions, respectively, and $z_{0}$ is an arbitrary $z$ value [22]. The derivation of the transfer matrix was straightforward, composed of the product of interface and boost matrices, and was explicitly shown in $[19,21]$. Note that the transfer matrix $\mathcal{T}$ depends only on $k_{x}$. Bloch states in SMIMs are indexed with $K$ in Equation (1), which determines the propagation characteristics for the $z$ axis; the explicit form is given by:

$$
i K d=\ln \{(\exp (i K d)\} \pm 2 \pi i m
$$

where $m$ is an integer and gives a branch only to the real part of the $K$. Thus, there remains the problem to determine a proper branch, which is examined later. Note that the Bloch band is independent of the value of $z_{0}$. At TM polarization, $F$ is the $y$ component of the magnetic field, whereas, at TE (transverse electric) polarization $(\mathbf{E} \| y), F$ is the $y$ component of the E-field. The transmission Bloch bands (blue and thin gray) in Figure $2 \mathrm{~d}$ were obtained under the exponentially non-growing condition for $\exp (i K d)$, such as $0 \leq \operatorname{Im}(K)<0.1$ and $0 \leq \operatorname{Im}(K)<1.0$, respectively; we call the former low-loss condition and the latter relatively large loss. Strictly, the Bloch bands in realistic SMIMs are quasi-transmission bands because optical loss is inevitable. This point is different from the Bloch bands in loss-free dielectric multilayer structures [18].

In Figure 2d, a feature in the Bloch band appears, for which the $k_{x}$ component widely supports the transmission Bloch band at about $3.0 \mathrm{eV}$ (double-ended arrow). The wide $k_{x}$ mode was recently employed to realize SR imaging with sub-50-nm resolution by implementing a hyperlens in an optical microscope [9]. It is also to be noted that a small $\operatorname{Im}\left(k_{z}\right)$ band shown with blue manifests itself as $\mathrm{T}$ peaks in Figure $2 b, c$. Thus, the Bloch state is well supported by the experimental data. 
(a)
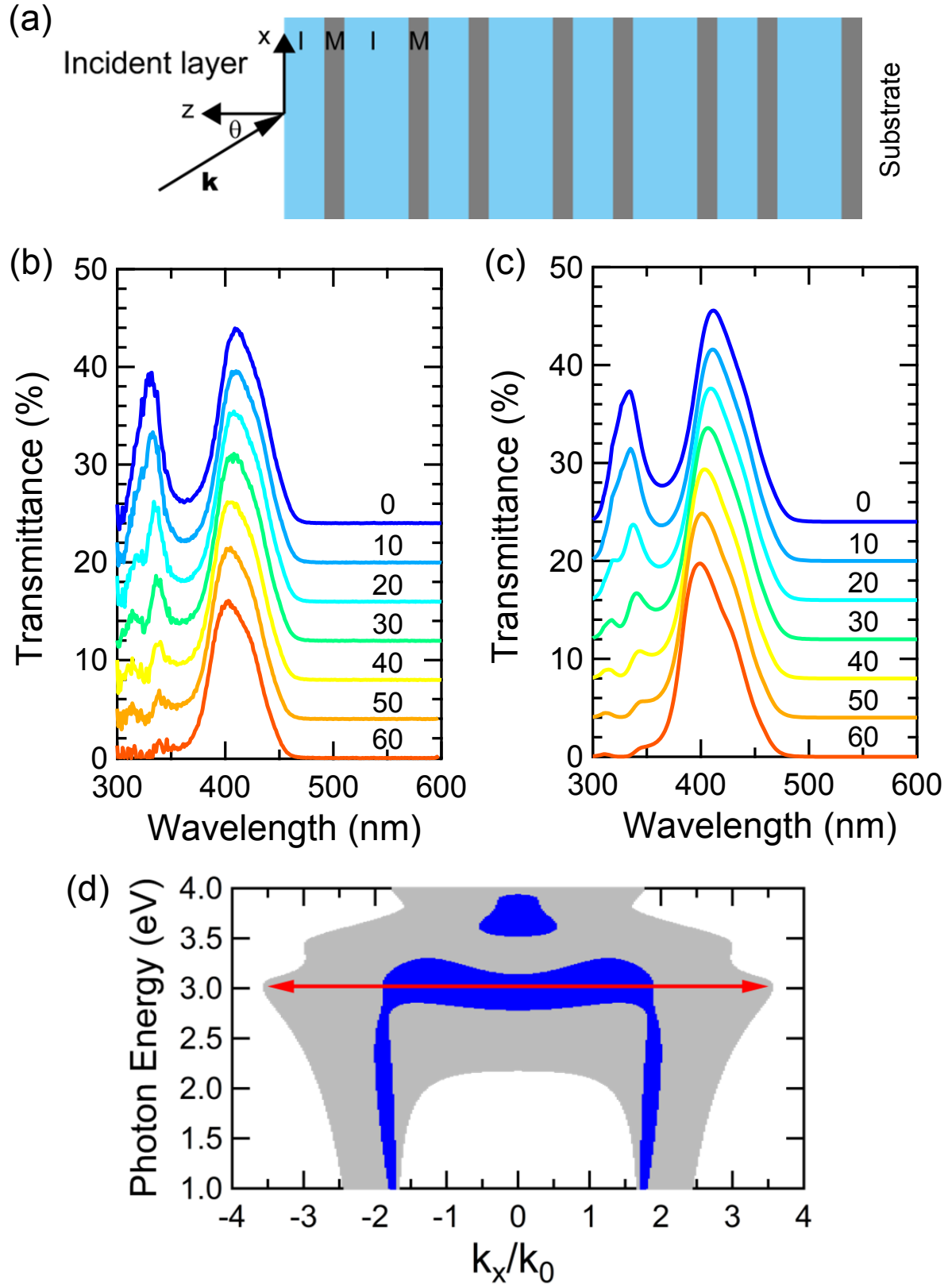

Figure 2. (a) Schematic of a SMIM of the four-layer unit cell, which has a transmission window at $410 \mathrm{~nm}$. (b,c) Measured and computed $\mathrm{T}$ spectra under $p$ polarization, respectively, which are shown with an offset for clarity. The incident angle varied from $0^{\circ}$ to $60^{\circ}$ at $10^{\circ}$ steps. (d) Transmission Bloch band at transverse magnetic (TM) polarization. Blue and light gray correspond to the low-loss and relatively large-loss bands described in the text, respectively. Double-ended arrows indicate the peak of transmission at $3.02 \mathrm{eV}$ (or $410 \mathrm{~nm})$.

\subsection{Six-Layer Unit Cell for Green Wavelengths}

Figure 3a schematically draws a SMIM of the six-layer unit cell of $\left(\mathrm{SiO}_{2} 51.2 \mathrm{~nm} / \mathrm{Ag} 14.7 \mathrm{~nm} / \mathrm{SiO}_{2}\right.$ $101.8 \mathrm{~nm} / \mathrm{Ag} 7.4 \mathrm{~nm} / \mathrm{SiO}_{2} 15.4 \mathrm{~nm} / \mathrm{Ag} 9.5 \mathrm{~nm}$ ). If the $\mathrm{SMIM}$ is a finite stacking, a $\mathrm{SiO}_{2}$ layer of 51.2 $\mathrm{nm}$ in thickness is set to touch the incident layer of air and a Ag layer of $9.5 \mathrm{~nm}$ to touch the $\mathrm{SiO}_{2}$ substrate. The unit cell was found through automatic search on a computer; the search started with 
the random generation of 30,000 unit cells of given periodicity and numbers of layers, selecting unit cells satisfying high $\mathrm{T}$ at $500 \mathrm{~nm}$ as independent as possible of the incident angles between $0^{\circ}$ and $80^{\circ}$ under $p$ polarization and then optimizing the best unit cell in the random generation. This automatic search is equivalent to a genetic algorithm search in the first generation; similar implementations to take optical quantity in MMs as the fitness were reported [28-30]. In the present search, fitness in the genetic algorithm was the sum of T evaluated at $10^{\circ}$-step incident angles from $0^{\circ}$ to $80^{\circ}$, and the constraint was the total thickness of the unit cell $(200 \mathrm{~nm})$ and the minimum thickness of each layer $(5$ $\mathrm{nm})$. Finite SMIMs, which were composed of four unit cells, were searched. The SMIM of the best unit cell exhibits T more than $30 \%$ at $500 \mathrm{~nm}$ and $\theta=0^{\circ}$, even when it contains 24 layers, half of which are metallic layers. We mention that the best-designed SMIM requires highly precise control of each layer; in practice, the ion-beam sputtering that we employed in Figure $2 \mathrm{~b}$ is able to meet this precision. This is because, although the growth rates of $\mathrm{Ag}$ and $\mathrm{SiO}_{2}$ were $0.31 \pm 0.03$ and $0.17 \pm 0.03 \mathrm{~nm} / \mathrm{s}$, respectively, the temporal control less than $0.3 \mathrm{~s}$ makes it practically possible to realize $0.1-\mathrm{nm}$ precision. Note again that the very good agreement of the experiment and theory in Figure 2 was obtained by the sputtering method and would not be obtained by other film-making methods.

Figure $3 \mathrm{~b}$ shows the transmission Bloch band of the SMIM of the six-layer unit cell at TM polarization, represented in a similar manner to Figure $2 \mathrm{~d}$. Figure $3 \mathrm{c}$ shows the equi-frequency TM-polarized contour at $2.4304 \mathrm{eV}$ (or $510.0 \mathrm{~nm}$ in wavelength); the real part of $k_{z}$ is shown with solid curves and the imaginary part with the dotted line. The real parts always contain both $+z-$ and $-z$-propagation components, shown with bold and thin curves, respectively. The equi-frequency contour is peculiar to TM polarization; at the low-loss range $\left(\left|k_{x}\right| \leq 1.65\right)$, the $\operatorname{Re}\left(k_{z}\right)$ takes positive values and forms a convex shape for the $+z$-propagation. At the other $k_{x}$ range $\left(\left|k_{x}\right|>1.65\right)$, the $\operatorname{Re}\left(k_{z}\right)$ becomes negative. The $\left(k_{x}, k_{z}\right)$ contour is far from the so-called hyperbolic shape; nevertheless, the mode serves as a hyper mode, as shown later. Note that the imaginary part is shown only for the $+z$-propagation component for simplicity.

Figure 3d shows the transmission Bloch band at TE polarization; red and light gray correspond to low-loss and relatively large-loss bands, respectively. Figure 3e shows the equi-frequency TE-polarized contour at $510.0 \mathrm{~nm}$; the real part of $k_{z}$ is shown with solid curves and the imaginary part with the dotted line. The real parts associated with $+z$ - and $-z$-propagation components are shown in a similar way to Figure 2c. The imaginary part is also shown similarly to Figure 2c. The equi-frequency contour is roughly spherical in shape around the origin, close to typical equi-frequency contours in insulators at non-resonant wavelengths.

Figure 4 shows the T spectra of the SMIM of the six-layer unit cell (Figure 3) in pseudo-color representation: (a) and (b) show TM and TE polarizations, respectively. To evaluate finite T, we set the SMIM to be 24-layers in total stacked on a $\mathrm{SiO}_{2}$ substrate. In this case, the SMIM had an 800-nm thickness in total. $\mathrm{A} \mathrm{SiO}_{2}$ layer of $51.2 \mathrm{~nm}$ in thickness was set to touch the incident layer of air. Incident angles varied from $-89^{\circ}$ to $89^{\circ}$ and, therefore, covered the $k_{x} / k_{0}$ range of $(-1,1)$ in Figure 3b,d. Evidently, the T peaks in Figure 4 agree with the low-loss Bloch band in Figure 3. Thus, the Bloch band is explicitly confirmed by the T spectra. 

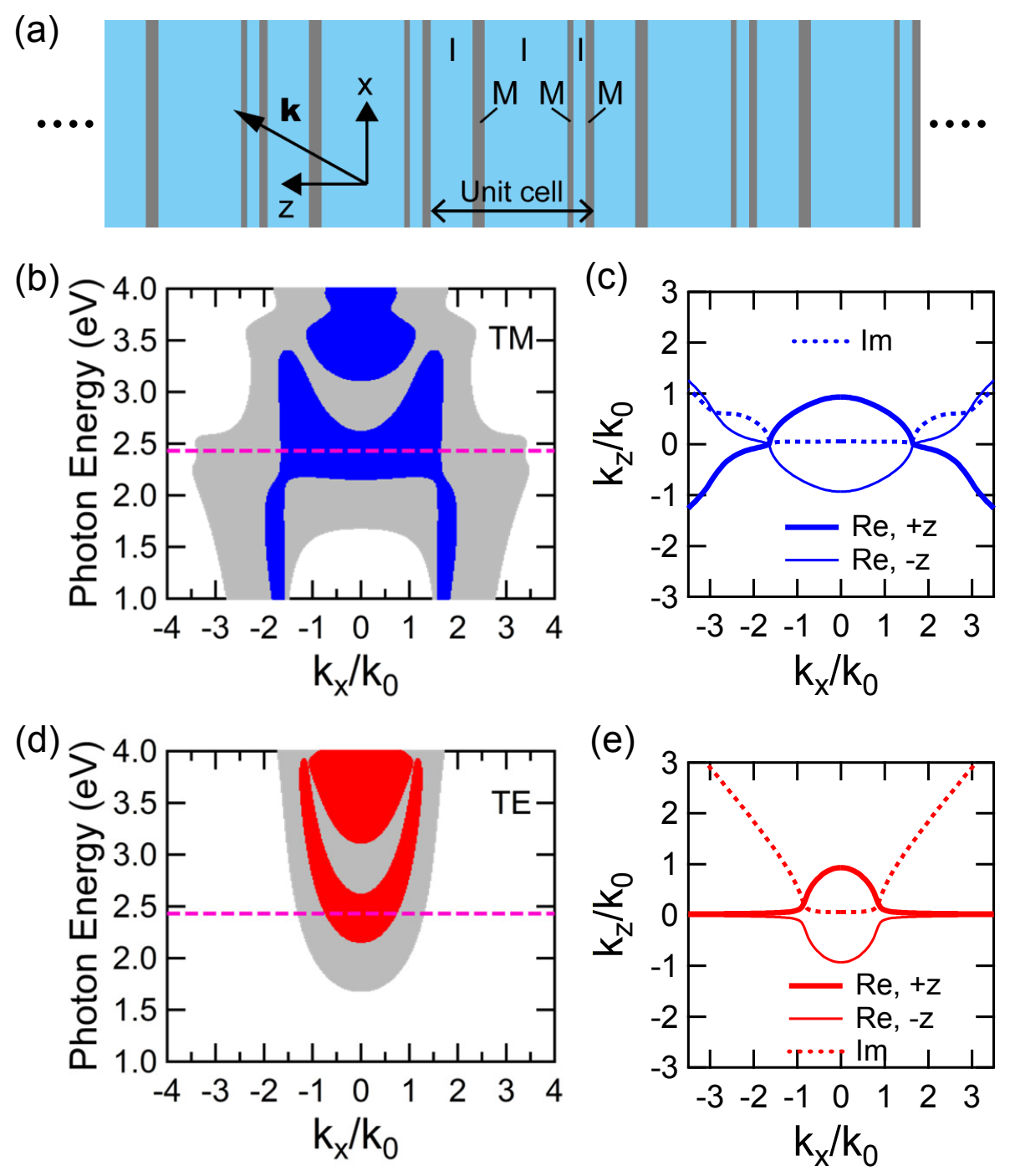

Figure 3. (a) Schematic of a SMIM of the six-layer unit cell that has a transmission window at $500 \mathrm{~nm}$. The structural parameters are described in the text. (b) Transverse magnetic (TM)-polarization Bloch band, shown in a similar manner to Figure 2d. (c) Equi-frequency TM contour at $2.4304 \mathrm{eV}$ (or $510.0 \mathrm{~nm}$ ). Solid lines denote the real part of $k_{z}$, and the dotted line the imaginary part. (d) TE-polarization Bloch band; red denotes the low-loss band and light gray the relatively large-loss band. (e) Equi-frequency TE contour at 510.0 $\mathrm{nm}$, represented similarly to (c).

As was referred to in Equation (2), it is necessary to determine a proper branch of Bloch-state index $K$, which is $k_{z}$ in Figure 3c,e. A direct way to determine the physical branch is shown in Figure 5. 
(a)

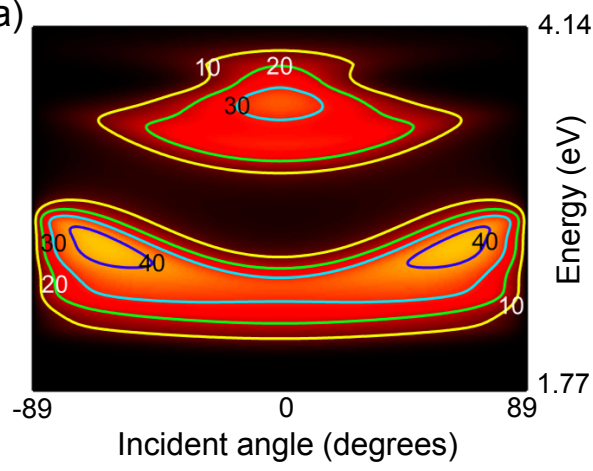

(b)

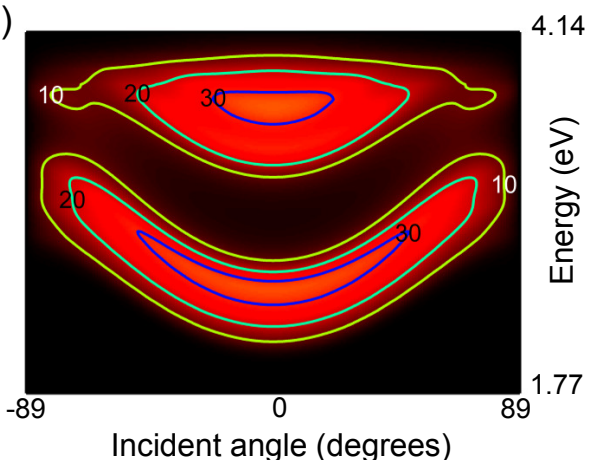

Figure 4. (a,b) 2D plots of T at TM and TE polarizations, respectively, represented with pseudo-color and contours.

(a) TM

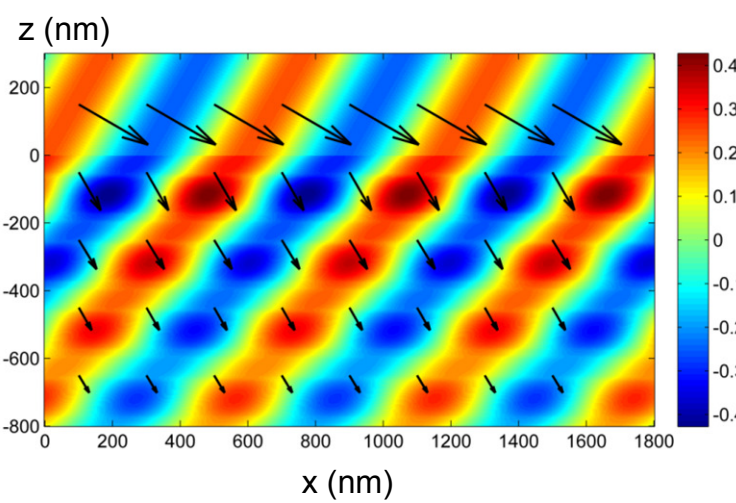

(b) TE

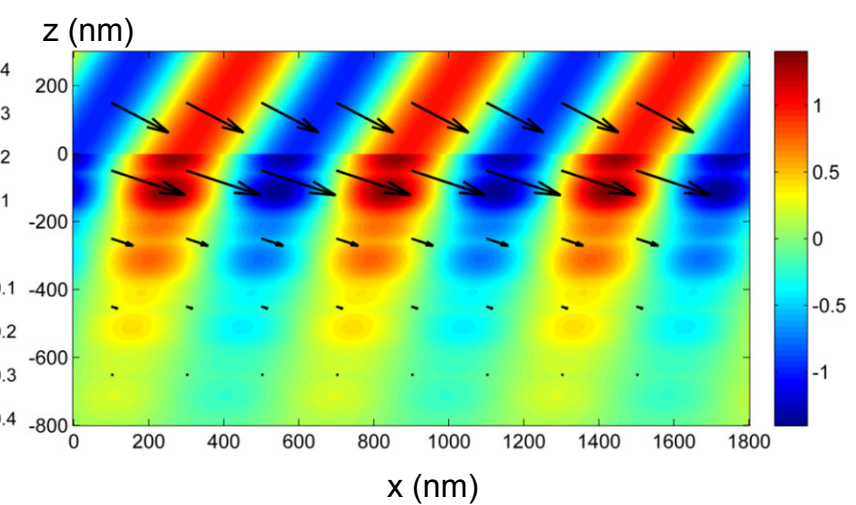

Figure 5. Illumination of the SMIM of the six-layer unit cell. (a,b) Electromagnetic (EM)-field distributions (color plots) at TM and TE polarizations, respectively. Specifically, the color plots represent snapshots of (a) $H_{y}$ and (b) $E_{y}$ components. The incident wavelength was $510.0 \mathrm{~nm}$, and the incident angle was set to $60^{\circ}$. Arrows denote Poynting vectors in the $x z$ plane.

Figure 5 shows EM-field distributions (color) and the Poynting vector (arrows) on the $x z$ plane at oblique incidence of $\theta=60^{\circ}$ in the illumination configuration of Figure 1. The color plots represent snapshots of $H_{y}$ and $E_{y}$ components in Figure 5a,b, respectively. Incident light of $510.0 \mathrm{~nm}$ in wavelength travels from the top and sheds on the surface of the SMIM of the six-layer unit cell at $z=0 \mathrm{~nm}$. Figure 5a,b corresponds to TM and TE polarizations, respectively, and accordingly display the $E_{x}$ and $E_{y}$ components. Note that, in the incident layer, we plotted only the incident component to clearly visualize the oblique incidence, omitting the reflection component. A proper branch in Equation (2) was determined as follows. The incident angle of $\theta=60^{\circ}$ means that the $k_{x}$ holds the relation of $k_{x} / k_{0}=\sqrt{3} / 2$, where $k_{x}$ is the $x$ component of wavevector in the SMIM and $k_{0}$ wavenumber of light in air. The wave front is formed in the SMIM, and refraction is observed, suggesting that refraction angle $\alpha$ at TM polarization is smaller than incident angle $\theta$ and refraction angle $\beta$ at TE polarization larger than $\theta$; thus we have a relation of $0^{\circ}<\alpha<\theta=60^{\circ}<\beta$, which implies that the $\left|k_{z}^{(\mathrm{TM})}\right|$ at TM polarization is larger than the $\left|k_{z}^{(\mathrm{TE})}\right|$ at TE polarization. Strictly, the E-field component in SMIMs contains both forward and backward components; therefore, the wave front does not necessarily provide 
a well-defined refraction angle. Still, in this case, the Poynting vector $\left(S_{x}, S_{z}\right)$ in Figure 4 is qualitatively consistent with the description of the refraction, supporting the validity. Finally, the $\operatorname{Re}\left(k_{z}\right)$ takes a positive value and satisfies with the relation of:

$$
\frac{\operatorname{Re}\left(k_{z}^{(\mathrm{TE})}\right)}{k_{0, z}}<1<\frac{\operatorname{Re}\left(k_{z}^{(\mathrm{TM})}\right)}{k_{0, z}}
$$

We thus reach a proper branch of $m=0$ in Equation (2), because any other $m$ does not meet Equation (3). The proper branch is shown in Figure $3 \mathrm{c}$ and e.

\subsection{EM-Field Images of a Deeply Subwavelength Object}

Figure 6a illustrates a configuration to test hyper mode in the SMIM of the six-layer unit cell. A Cr mask of a 25-nm slit and 23-nm thickness was set on the surface of a SMIM. Similarly to Figure 3a, the stacking direction of the layers is parallel to the $z$ axis.

Figure 6 b displays a computed $S_{z}$ distribution in the configuration of Figure 6a. The SMIM was introduced in Figure $3 \mathrm{a}$; the $S_{z}$ is represented with pseudo-color and contours. The $x$-polarized incident light of $S_{z}=-1$ was set to illuminate the Cr mask at the normal incidence. The wavelength was 510.0 $\mathrm{nm}$. Figure $6 \mathrm{c}$ is a reference to Figure $6 \mathrm{~b}$, presenting the $S_{z}$ distribution in the configuration where the SMIM was replaced with the $\mathrm{SiO}_{2}$ substrate. In comparison with Figure 6b,c, it was confirmed that diffraction is suppressed in the SMIM; the contour of $S_{z}=-0.04$ in Figure $6 \mathrm{~b}$ has a 94-nm width along the $x$ axis at the $z$ position close to the substrate, whereas the contour of $S_{z}=-0.06$ in the reference (Figure 6c) shows about a 600-nm width. Thus, the SMIM is able to keep the transferred image to a deeply subwavelength dimension. Such subwavelength EM-field distributions would be also useful for lithography. Actually, a SMIM was employed in interference lithography [31].

Figure 6d shows a computed $S_{z}$ distribution in a SMIM of a gain insulator. The structural parameters in the SMIM and the optical setup were the same as Figure 6b. The permittivity of the insulator $\varepsilon_{g}$ was set to $\varepsilon_{g}=2.1316-0.05 i$, which means a loss reduction with a small gain, compared to the loss compensation in other MMs reported so far [32,33]. We avoided introducing unrealistically extreme $\varepsilon_{g}$ values. As a result, the $S_{z}$ distribution inside the SMIM shows an improved diffraction-suppressing distribution, and the contour of $S_{z}=$ -0.08 reaches the substrate with a $120-\mathrm{nm}$ width along the $x$ axis. The EM fields in Figure 6 were calculated by rigorously coupled-wave analysis [34] combined with the S-matrix algorithm [26].

We here note the reason why the $S_{z}$ component was plotted in Figure 6. Simple E-field intensity $|\mathbf{E}|^{2}$ does not necessarily ensure the observable EM-field images, because the purely near-field component cannot be observed in the hyperlens microscopy observing a far-field component $[3,4]$. The $\left|S_{z}\right|$ component reaching the substrate is composed of a far-field component, proportional to $\left|E_{x}\right|^{2}$ in the configuration of Figure 6. Thus, the intensity distribution of the transmitted light is proportional to the $\left|S_{z}\right|$ distribution shown with the color plot. In the setting in Figure 6, when the value is $S_{z}=-0.06$, the intensity is locally $6 \%$ for the incident light intensity. The value is enough to observe the images. Note that the description using the $S_{z}$ component was already confirmed to work effectively in the experimental study in [9]. 
(a)

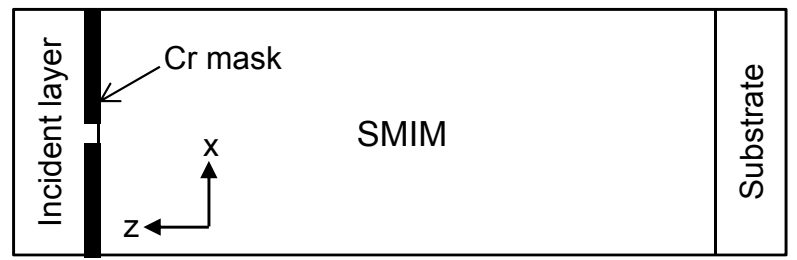

(b)

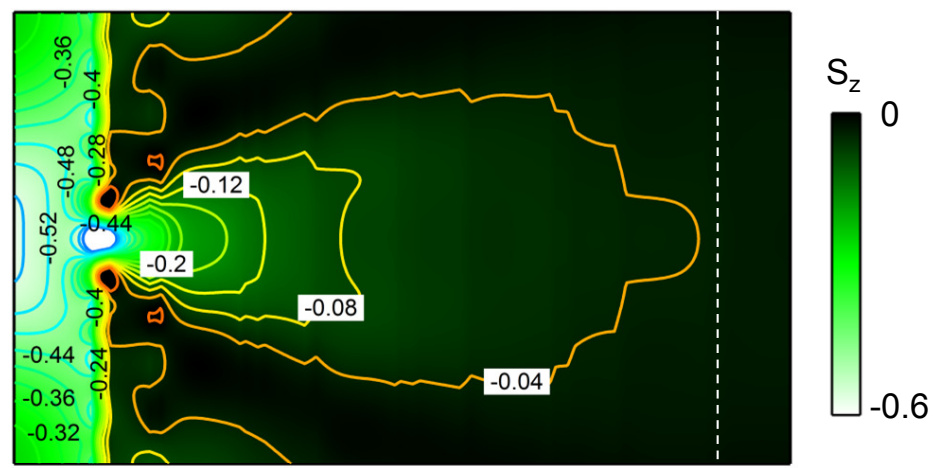

(c)

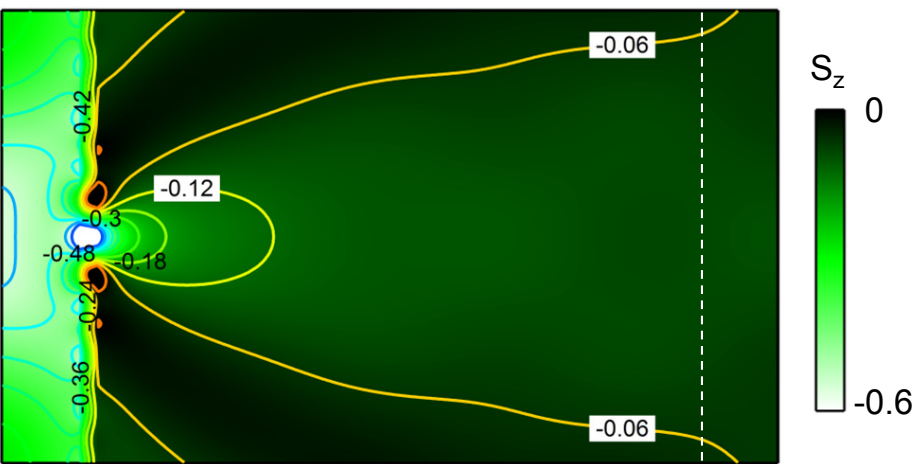

(d)

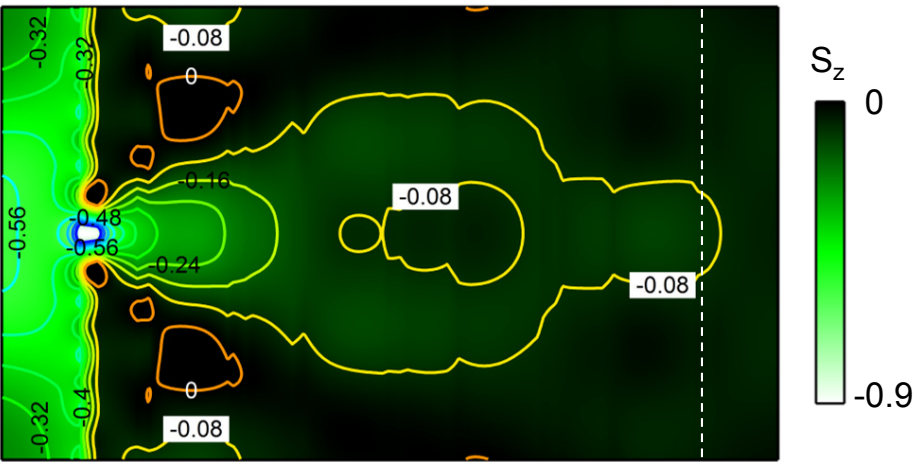

Figure 6. (a) Configuration of a SMIM with narrow Cr mask with a 25-nm slit. (b) Propagation of the deeply subwavelength object image via the hyper mode at $510.0 \mathrm{~nm}$ in the SMIM of six-layer unit cell. The $S_{z}$ component is shown with pseudo-color and contours. (c) Propagation in the $\mathrm{SiO}_{2}$ substrate after going through the $\mathrm{Cr}$ slit. (d) Propagation of the subwavelength object image in a SMIM, including the gain insulator of $\varepsilon_{g}=2.1316-0.05 i$. In (b) $-(d)$, the $x z$ axes are set similarly to (a); the Cr slit located at the half height in each panel is represented at the same dimensions to that in (a); and the positions of the SMIM/substrate interface are indicated by dashed lines in (b) and (d). 


\section{Discussion}

Let us examine the results and implications in Figures 2-5. The present Bloch-state analysis has advantages in: (i) the model-free or assumption-independent method, just starting from the fact that SMIMs are periodic structures; (ii) a good reproduction of experimental data, that is the good agreement of the transmission Bloch band with the T spectra; and (iii) being capable of describing the spatially dispersive behaviors of the equi-frequency contours. The last feature is absent in the effective medium model, usually assumed for SMIMs with local (or k-independent) effective parameters, such as $\varepsilon_{\|}$and $\varepsilon_{\perp}$. However, as shown in Figure 3, the equi-frequency contours are peculiar and not simply analytical in shape, associated with anisotropic absorption. Therefore, the effective medium model cannot describe them. We point out that, if one introduces the k-dependent $\varepsilon_{\|}$and $\varepsilon_{\perp}$ and extends the effective medium model, the upgraded model would be equivalent to this Bloch-state analysis; such a model was proposed [12], but has not been applied to arbitrary k. The upgraded model would have, at least, two parameters $\left(\varepsilon_{\|}(\mathbf{k})\right.$ and $\left.\varepsilon_{\perp}(\mathbf{k})\right)$ and be more complicated than the present analysis that has been conducted in a thorough way.

(a)

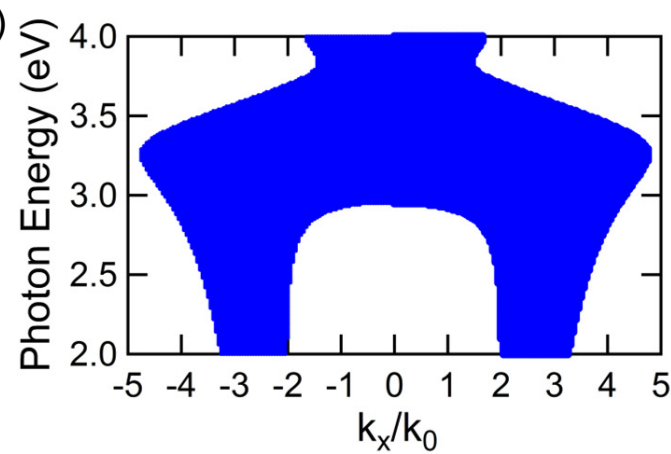

(c)

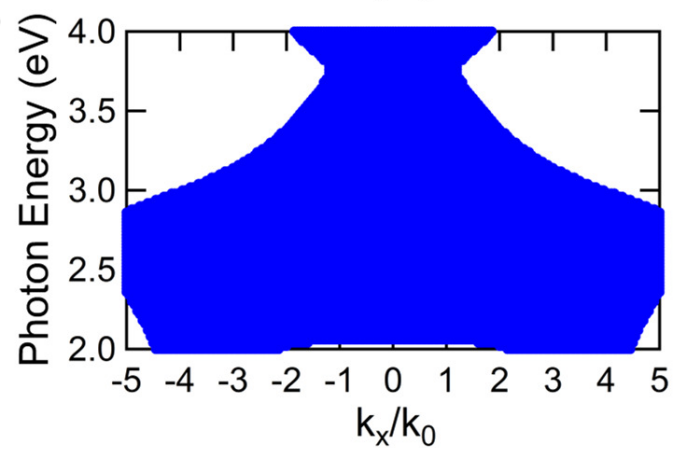

(b)

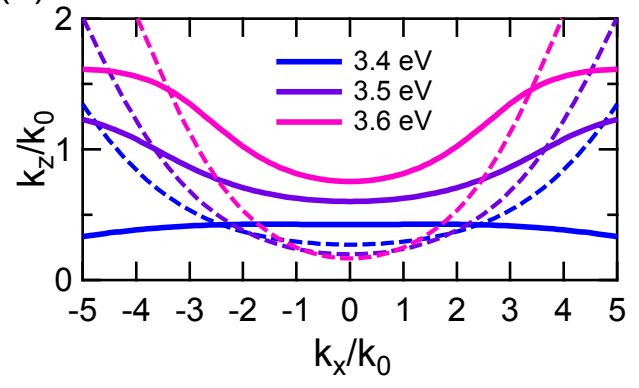

(d)

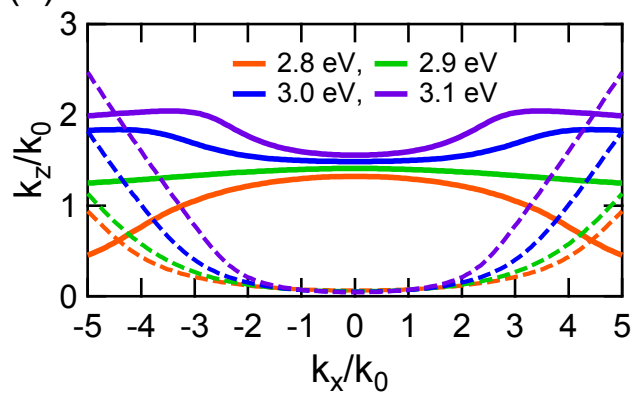

Figure 7. Other SMIMs reported to date. (a) TM-polarized transmission Bloch band of a SMIM of the $\left(\mathrm{Ag} 30 \mathrm{~nm} / \mathrm{SiO}_{2} 30 \mathrm{~nm}\right.$ ) unit cell (blue). The band was extracted under the relatively large-loss condition. (b) Equi-frequency contours at 3.4 (blue), 3.5 (purple) and $3.6 \mathrm{eV}$ (light purple) in (a), which appear from the bottom to the top. Solid lines denote the real part and dashed lines the imaginary part. (c) TM-polarized transmission Bloch band of SMIM of the (Ag $30 \mathrm{~nm} /$ insulator (I) $50 \mathrm{~nm}$ ) unit cell, where I has a permittivity of 4.84 . The band was extracted similarly to (a). (d) Equi-frequency contours at 2.8 (orange), 2.9 (green), 3.0 (blue) and $3.1 \mathrm{eV}$ (purple) in (c), which appear from the bottom to the top. The real and imaginary parts are shown in a similar way to (b). 
We here refer to the results of the Bloch-state analysis of the typical SMIMs employed in experiments to date. Figure 7 shows the transmission Bloch bands at TM polarization (blue color) and equi-frequency contours.

A typical SMIM comprises the unit cell of $\left(\mathrm{Ag} 30 \mathrm{~nm} / \mathrm{SiO}_{2} 30 \mathrm{~nm}\right)$ and has a transmission band in the ultraviolet range; similar SMIMs were employed in [4,6,7]. Figure 7a,b exhibits the Bloch-state analysis for the SMIM of one-to-one thickness in the metal and insulator layers. The $k_{x}$-wide Bloch band appears in the UV range around $3.3 \mathrm{eV}$ (Figure 7a).

The Bloch band was extracted under a relatively large-loss condition. Figure $7 \mathrm{~b}$ shows equi-frequency contours at 3.4 (blue curve), 3.5 (purple curve) and 3.6 eV (light purple curve); solid and dashed curves denote the real and imaginary parts, respectively. Obviously, the contours change shape as the photon energy increases, deforming from the quite flat to concave shapes around $k_{x}=0$ at 3.4 to $3.6 \mathrm{eV}$. On the other hand, the optical loss or $\operatorname{Im}\left(k_{z}\right)$ increases as $\left|k_{x}\right|$ gets larger, which is an explicit signature of spatial dispersion; simultaneously, the contour at $3.6 \mathrm{eV}$ deviates from the concave shape to a convex shape at $\left|k_{x}\right|>3$. In short, the hyperbolic dispersion assumed in the previous theoretical reports does not exist, even in the typical SMIM. These results are consistent with the feature that SMIMs are spatial dispersive, being out of scope for the effective medium model.

Figure 7c,d is the result for the SMIM of the $(\mathrm{Ag} 30 \mathrm{~nm} / \mathrm{I} 50 \mathrm{~nm})$-unit cell where the I was assumed to have a permittivity of 4.84 , which was set considering a high-refractive-index insulator, such $\operatorname{as} \mathrm{Ta}_{2} \mathrm{O}_{5}$. Similar designs were recently reported $[8,10,11]$. The $k_{x}$-wide band is located around $2.8 \mathrm{eV}$ and moves to lower energy than the band in Figure $7 \mathrm{a}$. The Bloch band was extracted similarly to that in Figure 7a. The equi-frequency contours at 2.8 to $3.1 \mathrm{eV}$ are shown in Figure $7 \mathrm{~d}$, appearing in the order of bottom-to-top. The real (solid curves) and imaginary parts (dashed curves) exhibit a similar profile to those in Figure $7 \mathrm{~b}$. Overall, the dispersions indicate spatial dispersion and non-hyperbolic dispersion in the typical SMIMs reported so far.

In contrast to the conventional descriptions claiming hyperbolic dispersion, the present Bloch analysis in Figure 7 explicitly shows that propagation modes in the typical SMIMs in experiments are not well approximated by hyperbolic dispersion. Instead, non-trivial dispersions were derived, associated with the imaginary part (i.e., optical loss).

\section{Conclusions}

We have proposed a concrete design of a SMIM that serves as an SR-imaging material at green wavelengths. The design assumed only $\mathrm{Ag}$ and a low refractive index common insulator, such as $\mathrm{SiO}_{2}$, and presented the well-designed six-layer unit cell. The Bloch-state analysis, which reproduces the experimental data and is able to handle SMIMs of complex unit cells, clarified that the SMIM has hyper modes, suppressing diffraction. We also showed that the loss reduction with a small gain in the insulator can improve the SR-imaging capability. The present method, which provides a firm basis to describe SMIMs, will serve quantitative designs of unexplored SMIMs and precise evaluations of the optical performance. 


\section{Acknowledgments}

This study was partially supported by the JST (Japan Science and Technology Agency) PRESTO program and by the Konica Minolta Imaging Science Award. Numerical implementations were supported by Cyberscience Center, Tohoku University, through HPCI (High Performance Computing Infrastructure) System Research Project (ID hp140068).

\section{Conflicts of Interest}

The author declares no conflict of interest.

\section{References}

1. Pendry, J.B. Negative refraction makes a perfect lens. Phys. Rev. Lett. 2000, 85, 3966-3969.

2. Ramakrishna, S.A.; Pendry, J.B. Removal of absorption and increase in resolution in a near-field lens via optical gain. Phys. Rev. B 2003, 67, 201101.

3. Salandrino, A.; Engheta, N. Far-field subdiffraction optical microscopy using metamaterial crystals: Theory and simulations. Phys. Rev. B 2006, 74, 075103.

4. Jacob, Z.; Alekseyev, L.V.; Narimanov, E. Optical hyperlens: Far-field imaging beyond the diffraction limit. Opt. Express 2006, 14, 8247-8256.

5. Fang, N.; Lee, H.; Sun, C.; Zhang, X. Sub-diffraction-limited optical imaging with a silver superlens. Science 2005, 308, 534-537.

6. Liu, Z.; Lee, H.; Xiong, Y.; Sun, C.; Zhang, X. Far-field optical hyperlens magnifying sub-diffraction-limited objects. Science 2007, 315, 1686.

7. Smolyaninov, I.I.; Hung, Y.-J.; Davis, C.C. Magnifying superlens in the visible frequency range. Science 2007, 315, 1699-1701.

8. Rho, J.; Ye, Z.; Xiong, Y.; Yin, X.; Liu, Z.; Choi, H.; Bartal, G.; Zhang, X. Spherical hyperlens for two-dimensional sub-diffractional imaging at visible frequencies. Nat. Commun. 2010, 1, 143.

9. Iwanaga, M. Hyperlens-array-implemented optical microscopy. Appl. Phys. Lett. 2014, 105, 053112.

10. Verhagen, E.; de Waele, R.; Kuipers, L.; Polman, A. Three-dimensional negative index of refraction at optical frequencies by coupling plasmonic waveguides. Phys. Rev. Lett. 2010, 105, 223901.

11. Xu, T.; Agrawal, A.; Abashin, M.; Chau, K.J.; Lezec, H.J. All-angle negative refraction and active flat lensing of ultraviolet light. Nature 2013, 497, 470-474.

12. Elser, J.; Podolskiy, V.A.; Salakhutdinov, I.; Avrutsky, I. Nonlocal effects in effective-medium response of nanolayered metamaterials. Appl. Phys. Lett. 2007, 90, 191109.

13. Kidwai, O.; Zhukovsky, S.V.; Sipe, J.E. Effective-medium approach to planar multilayer hyperbolic metamaterials: Strengths and limitations. Phys. Rev. A 2012, 85, 053842.

14. Cortes, C.L.; Newman, W.; Molesky, S.; Jacob, Z. Quantum nanophotonics using hyperbolic metamaterials. J. Opt. 2012, 14, 063001.

15. Drachev, V.P.; Podolskiy, V.A.; Kildishev, A.V. Hyperbolic metamaterials: new physics behind a classical problem. Opt. Express 2013, 21, 15048-15064. 
16. Poddubny, A.; Iorsh, I.; Belov, P.; Kivshar, Y. Hyperbolic metamaterials. Nat. Photonics 2013, 7, 958-967.

17. Iwanaga, M. Effective optical constants in stratified metal-dielectric metameterial. Opt. Lett. 2007, 32, 1314-1316.

18. Yeh, P.; Yariv, A.; Hong, C.-S. Electromagnetic propagation in periodic stratified media. I. General theory. J. Opt. Soc. Am. 1977, 67, 423-438.

19. Markoš, P.; Soukoulis, C. M. Wave Propagation From Electrons to Photonic Crystals and Left-Handed Materials, (Princeton Univ. Press, Princeton, 2008).

20. Schilling, J. Uniaxial metallo-dielectric metamaterials with scalar positive permeability. Phys. Rev. E 2006, 74, 046618.

21. Iwanaga, M. Diversity of optical indices in stratified metal-dielectric metamaterials. Proc. SPIE 2009, 7395, 73951L.

22. Iwanaga, M. Photonic metamaterials: a new class of materials for manipulating light waves. Sci. Technol. Adv. Mater. 2012, 13, 053002.

23. Zhukovsky, S.V.; Kidwai, O.; Sipe, J.E. Physical nature of volume plasmon polaritons in hyperbolic metamaterials. Opt. Express 2013, 21, 14982-14987.

24. Zhukovsky, S.V.; Orlov, A.A.; Babicheva, V. E.; Lavrinenko, A.V.; Sipe, J.E. Photonic-band-gap engineering for volume plasmon polaritons in multiscale multilayer hyperbolic metamaterials. Phys. Rev. A 2014, 90, 013801.

25. Orlov, A.A.; Krylova, A.K.; Zhukovsky, S.V.; Babicheva, V.E.; Belov, P.A. Multiperiodicity in plasmonic multilayers: General description and diversity of topologies. Phys. Rev. A 2014, 90, 013812.

26. Li, L. Formulation and comparison of two recursive matrix algorithm for modeling layered diffraction gratings. J. Opt. Soc. Am. A 1996, 13, 1024-1035.

27. Rakić, A.D.; Djurušić, A.B.; Elazar, J.M.; Majewski, M.L. Optical properties of metallic films for vertical-cavity optoelectronic devices. Appl. Opt. 1998, 37, 5271-5283.

28. Kildishev, A.V.; Chettiar, U.K.; Liu, Z.; Shalaev, V. M.; Kwon, D.-H.; Bayraktar, Z.; Werner, D.H. Stochastic optimization of low-loss optical negative-index metamaterial. J. Opt. Soc. Am. B 2007, 24, A34-A39.

29. Chen, P.Y.; Chen, C.H.; Wang, H.; Tsai, J.H.; Ni, W.X. Synthesis design of artificial magnetic metamaterials using a genetic algorithm. Opt. Express 2008, 16, 12806-12818.

30. Iwanaga, M. Optically deep asymmetric one-dimensional metallic grooves: Genetic algorithm approach. J. Opt. Soc. Am. B 2009, 26, 1111-1118.

31. Ishii, S.; Kildishev, A.V.; Narimanov, E.; Shalaev, V.M.; Drachev, V.P. Sub-wavelength interference pattern from volume plasmon polaritons in a hyperbolic medium. Laser Photon. Rev. 2013, 7 , 265-271 (2013).

32. Xiao, S.; Drachev, V.P.; Kildishev, A.V.; Ni, X.; Chettiar, U.K.; Yuan, H.-K.; Shalaev, V. M. Loss-free and active optical negative-index metamaterials. Nature 2010, 466, 735-738.

33. Wuestner, S.; Pusch, A.; Tsakmakidis, K.L.; Hamm, J.M.; Hess, O. Overcoming losses with gain in a negative refractive index metamaterial. Phys. Rev. Lett. 2010, 105, 127401. 
34. Li, L. New formulation of the Fourier modal method for crossed surface-relief gratings. J. Opt. Soc. Am. A 1997, 14, 2758-2767.

(C) 2015 by the author; licensee MDPI, Basel, Switzerland. This article is an open access article distributed under the terms and conditions of the Creative Commons Attribution license (http://creativecommons.org/licenses/by/4.0/). 